\title{
Tagungsbericht
}

\section{Public Administration and Private Enterprise: Co-operation, Competition and Regulation}

\author{
Bericht über die Tagung des International Institute of Administrative Sciences (IIAS) und der Deutschen Sektion des IIAS \\ vom 20. bis 23. September 2005 in Berlin
}

Welche Möglichkeit haben Staaten, sich im Zeichen sinkender Einnahmen ihre Handlungsfähigkeit zu bewahren? Die Forderung nach einer gezielten Aufgabenkritik und die Suche nach Kooperationsformen zwischen öffentlichen und privaten Akteuren beherrscht derzeit die Diskussion. Öffentlich-Private Partnerschaften werden in Deutschland mit großen Erwartungen betrachtet, die sich oftmals mehr auf die nahe liegenden Chancen als auf die - verdeckten - Risiken öffentlich-privater Leistungserbringung konzentrieren. Ein internationaler Vergleich kann ein Mittel dazu sein, »blinde Flecken der einzelnen Organisationsformen aufzudecken und zugleich Anregungen für mögliche Modelle liefern - und zwar sowohl innerhalb der legislatorischen Rahmenbedingungen als auch unter deren Fortentwicklung.

Die Frage, wie Interaktionsformen zwischen dem öffentlichen und dem privaten Sektor organisiert werden können und welche Mindestanforderungen Staaten, etwa bei der Sicherstellung fairer Marktbedingungen, zu gewährleisten haben, war Gegenstand der 3rd Specialised International Conference des Internationalen Instituts für Verwaltungswissenschaften (IIAS) und seiner Deutschen Sektion, die unter dem Titel »Public Administration and Private Enterprise: Co-operation, Competition and Regulation« vom 20. bis 23. September 2005 in Berlin stattfand.* Die Konferenz widmete sich dieser Thematik im Rahmen von vier Workshops. Der Präsident des IIAS, Prof. Dr. Franz Strehl, der Präsident der Deutschen Sektion, Prof. Dr. Hans Peter Bull, der Staatssekretär im Bundesinnenministerium, Lutz Diwell, und Prof. Dr. Luiz Carlos Bresser-Pereira (Getúlia Vargas Foundation, Sao Paulo, Brasilien, als Gesamtberichterstatter) sowie der Generaldirektor des IIAS, Michael Duggett, führten in das Tagungsthema ein. Die Keynote Speech zum Thema »Successful Public-Private-Partnership in the Field of ICT-Services: joint venture between the German Aerospace Center (DLR) and T-Systems« wurde von Dr. Stephan Witteler (Vorstandsmitglied bei T-Systems) gehalten.

Die Analyse der Wirkungsvoraussetzungen und der Grenzen Öffentlich-Privater Partnerschaften stand im Mittelpunkt des ersten Workshops unter der Leitung von Prof. Dr. Ian Thynne (Charles Darwin University, Australien). Unter der leitenden Fragestellung nach »Co-operation and Partnership of Public Administration and Private Enterprise: economic opportunities, constitutional ramifications and political objections? « wurden Beispiele für die Ausgestaltung von Public Private Partnership-Projekten in verschiedenen politischen Systemen vorgestellt, die mit spezifischen Problemlagen bei der Ausgestaltung dieser Kooperationen zwischen dem staatlichen und dem privaten Sektor einhergingen. Die Vorträge betrafen ebenso demokratische Marktgesellschaften in den unterschiedlichsten Ausprägungen wie auch Transformationsgesellschaften in Osteuropa oder in Asien und Afrika und schließlich sozialistische Staaten wie China, die jeweils sehr unterschiedliche Rahmenbedingungen für Kooperationsformen zwischen staatlichen und privaten Akteuren aufweisen. Daran anschließend versuchte der Workshop, Typisierungen bestimmter Organisationen, Güter und Dienstleistungen zu bilden, welche am ehesten für derartige Interaktionsformen in Betracht kommen. Diese Fragen führten angesichts der Vielzahl der behandelten Systeme auch immer wieder in politologische und (verfassungs-) rechtliche Bereiche hinein.

Der zweite Workshop befasste sich mit dem Gesamtthema unter dem Aspekt der Regulierung. Geleitet von der Frage »Regulation: the concept and its enforcement. Experiences made by regulatory agencies in different states « widmete sich der Workshop unter der Leitung des Sektionspräsidenten beim französischen Staatsrat, $\mathrm{Mi}$ chel Franc, der Suche nach den gemeinsamen Charakteristika von »Regulierung «, welche erneut unter sehr unterschiedlichen institutionellen Rahmenbedingungen aus den beschriebenen unterschiedlichen politischen Systemen in den Blick genommen wurde. Jenseits der politischen Umweltfaktoren versuchte der Workshop, »konstante« Wirkungsvoraussetzungen für erfolgreiche Regulierungen wie die Etablierung unabhängiger Behörden mit festen Etats und einer unparteiischen Bewerberauswahl festzustellen, was jedoch angesichts der Vielzahl der unterschiedlichen Modelle ebenfalls durchaus ambitioniert war.

Mit dem Bereich des Öffentlichen Sektors beschäftigte sich der dritte Workshop unter der Leitung der Heidelberger Oberbürgermeisterin Beate Weber. Die Vorträge unter dem Gesamttitel »Competition, Co-operation and Conflict in the Field of Public Services«. Dieser Workshop untersuchte die unterschiedlichen Möglichkeiten von Kommunen, sich Privater bei der Erbringung ihrer Aufgaben zu bedienen. Insbesondere die Chancen und Grenzen der Bildung interner »Märkte« für Verwaltungsleistungen wurde in diesem Rahmen analysiert. Den Blick über nationale Regelungen hinaus erhob schließlich der vierte Workshop unter der Leitung von Claudius Bamidele Olowu, Principal Governance Adviser bei der African Development Bank in Tunis. Die Leitfrage nach den $»$ Regulatory Powers of National Governments in a Globalized Economy« eröffnete den Diskurs über die Möglichkeiten nationaler Staaten, regulierend auf die international verflochtene Wirtschaft einzuwirken.

Neben den Workshops zum Generalthema widmeten sich auch einige spezielle Podiumsdiskussionen dem Thema. Unter der Leitung der südafrikanischen Ministerin Geraldine Fraser Moleketi wurden Aspekte von Global Governance auch in einer Podiumsdiskussion vertieft. Das UN Panel unter der Co-Moderation von Guido Berbucci (Direktor bei DPADM/UNDESA, Vereinte Nationen) und Michael Duggett (Generaldirektor des IIAS) schließlich untersuchte die Frage nach einer »Public Private Partnership for Economic and Social Development $\ll$.

Spezielle Podiumsdiskussionen beschäftigten sich mit der Situation in einzelnen Regionen. Das German Panel nahm die in Deutschland derzeit besonders intensiv geführte Diskussion um eine Reform des Öffentlichen Dienstes in den Blick. Unter Moderation von Prof. Dr. Werner Jann (Universität Potsdam) diskutierten der Staatssekretär im Bundesinnenministerium, Dr. Göttrik Wewer, sowie Prof. Dr. Dieter Schimanke (Staatssekretär a. D.) und der stellvertretende Dekan der Hertie School of Governance, Dr. Jobst Fiedler, mit zwei Vertreterinnen aus der Wirtschaft, Dorothee Belz von der Microsoft Deutschland GmbH und Katrin Schütz vom Bund der Wirtschaftsjunioren, über »Administrative Reform and the Reduction of $\mathrm{Bu}$ reaucracy: An Ongoing Challenge for Public Administration and Private Enterprise«. Die Frage nach den »North American Approaches and Methods of Co-operation between Public Administration and Private Enterprise« untersuchte das von Prof. Dr. Steve Kelman (Harvard University) geleitete North American Panel. Das Asia Pacific Panel, das von Prof. Hiromitsu Kataoka (Waseda University, Japan) und von Prof. Itoko Suzuki (Ritsumeikan Asia-pacific University, Japan) geleitet wurde, widmete sich der Analyse des »Contracting-out in Local Governments: outcomes, limits and challenges «.

Das Spektrum der Themen zeigt, dass der Begriff der Public Private Partnership - insbesondere im internationalen Vergleich - recht vielgestaltig ist. So kam es in den Workshops auch oft zu interessanten Diskussionen über vergleichbare Rechtsformen, die je nach Land unterschiedliche Bezeichnungen erfuhren - und umgekehrt über unterschiedliche Rechtsformen, die sämtlich als Public Private Partnership bezeichnet wurden. Besonders spannend wurde die Diskussion immer dann, wenn über konkrete Projekte und konkrete Rechts- und Organisationsfragen diskutiert wurde, da dann die einzelnen Möglichkeiten nach nationalem Recht konkretisiert und international vergleichbar wurden. Umgekehrt entwickelten sich gerade im vierten Workshop oftmals theoretisch interessante Debatten, die sich mit den Möglichkeiten eines »dritten Wegs « zwischen ausschließlich staatlich und ausschließlich privat organisierter Regelung staatenübergreifender Fragen, etwa im Bereich des Umweltschutzes, beschäftigten.

* Das Konferenzprogramm ist abrufbar unter: http://www.iias-berlin-2005.de/. 


\section{Tagungsbericht}

Besondere Aufmerksamkeit fand die Vorstellung eines Historischen Projekts anlässlich des 75. Jubiläums des Internationalen Instituts für Verwaltungswissenschaften. Prof. Dr. Fabio Rugge (Pavia), Prof. Dr. Stefan Fisch (Speyer), Prof. Dr. Gavin R. Drewry (Royal Holloway University, U.K.) und Prof. Dr. Jean-Marie Yante stellten einen soeben erschienenen Band zur Geschichte des Internationalen Instituts (Fabio Rugge/Michael Duggett (eds.), IIAS 1930-2005, IOS Press, Amsterdam et al. 2005) vor. Dabei beleuchtete der Beitrag von Prof. Dr. Stefan Fisch über »Origins and History of the International Institute of Administrative Sciences: From Its Beginnings to Its Reconstruction After World War II (1910-1944/47)« die Geschichte des Internationalen Instituts, das aus der Idee der Selbstorganisation urbanistischer Aktivitäten entstand, und damit eng dem Tagungsthema verbunden war.

Die traditionelle Braibant Lecture wurde vom früheren Präsidenten des Internationalen Instituts, Prof. Dr. Ignazio Pichardo Pagaza (Mexico) zum Thema »Public Administration and Environment Protection in Latin America « gehalten. Der Präsident des IIAS, Prof. Dr. Franz Strehl, moderierte die Diskussion, an die sich ein Empfang auf Einladung des Internationalen Instituts anschloss.

Die Tagung in der Mitte Berlins wurde von einem Rahmenprogramm begleitet, aus welchem nur einige besonders eindrückliche Ereignisse erwähnt seien. Der feierliche Willkommensempfang nach der Eröffnung der Tagung fand auf Einladung des Bundesinnenmini- steriums und der Deutschen Bank in den Räumlichkeiten der Deutschen Bank AG an historischer Stätte in Berlin-Mitte statt. Besonderen Anklang fand der letzte Abend, welcher die Konferenzteilnehmer auf einen Spargel- und Kürbishof im brandenburgischen Klaistow entführte. Der dortige freundliche Empfang in zünftiger Umgebung auf Einladung der Deutschen Sektion des Internationalen Instituts spiegelte die angenehm unprätentiöse und zugleich präzise Gesamtorganisation der Konferenz durch das Organisationskomitee der Deutschen Sektion, Prof. Dr. Hans Peter Bull (Universität Hamburg), Dr. Christoph Hauschild (Bundesministerium des Innern, Berlin) und Claudia Schiefelbein (Universität Potsdam) wider. Die nächste Konferenz des IIAS wird im Jahre 2006 in Monterrey/Mexiko stattfinden.

Es bleibt aus der Konferenz der Gesamteindruck, dass die Frage der Kooperationen zwischen privatem und öffentlichem Sektor stets die Frage nach dem Selbstverständnis der staatlichen Tätigkeit aufwirft. Versteht sich der Staat als planend oder als Rahmen setzend oder will er entwicklungssteuernd auf die Umweltfaktoren einwirken? Erst nach einer solchen Grundentscheidung lassen sich taugliche Modelle von öffentlich-privaten Kooperationsformen entwickeln, welche nicht ubiquitär einsetzbar sind. Dennoch boten die vorgestellten internationalen Modelle Denkanstöße für die deutsche Debatte über ein Verfahrenskooperationsrecht, denen im Weiteren näher nachzugehen sein wird.

Dr. Margrit Seckelmann, Speyer

\section{Nachrichten}

\section{Baulücken im Internet}

Im Düsseldorfer Stadtgebiet gibt es 545 Baulücken, die ein beachtliches Potenzial für den Wohnungsbau darstellen. Nach Feststellungen des städtischen Planungsamtes ließen sich hier bis zu 4.000 Wohnungen errichten. Um diese Flächen in den Blickpunkt möglicher Interessenten zu rücken, haben die Stadtplaner alle Baulücken detailliert erfasst und im Internetangebot der Stadt Düsseldorf unter www.duesseldorf.de/planung veröffentlicht. Neben einem Foto des Grundstückes sind auch Lagepläne und Hinweise zur baurechtlichen Situation dargestellt. Die Landeshauptstadt weist als eine der ersten Städte in Deutschland ihr Baulandkataster, bestehend aus Baugebieten und Baulücken, im Internet aus. $\mathrm{Zu}$ den bereits veröffentlichten Baugebieten für den Wohnungsbau sind jetzt noch die Baulücken hinzu gekommen.

Für Rückfragen wenden Sie sich bitte an das Amt für Kommunikation, Telefon: (0211) 89-93131.

\section{Papierloser Landtag Steiermark}

Mit der konstituierenden Sitzung des Steiermärkischen Landtages und der Wahl des Landeshauptmannes Mag. Franz Voves wurde PALLAST erfolgreich in Betrieb genommen. Dies ist der europaweit erste Einsatz eines Gesetzgebungs-Workflow für Parlamente mit digitaler Signatur. Der Schriftverkehr zwischen Abgeordneten, Landtagsklubs und Landtagsverwaltung erfolgt ab sofort elektronisch. Alle Abgeordneten verwenden dazu einen Laptop und eine persönliche Signaturkarte (mit der Bürgerkarte als USB-Stick).

Die Vorbereitung von Anträgen und Sitzungen erfolgt online mit PALLAST. Sind Unterschriften weiterer Abgeordneter für bestimmte Prozesse notwendig, werden diese elektronisch angefordert. Über die Protokollierung ist jederzeit nachvollziehbar, wer wann Änderungen am Dokument vorgenommen hat. So hilft PALLAST durch Vorlagen und Regeln, formale Fehler zu vermeiden. Diese Sicherheit und Vereinfachung sind neben der Kostenreduktion die wichtigsten Gründe für die Innovation.

Weitere Informationen: icomedias Gmbh, Mag. Heidemarie Simon, Telefon: +43-316-721671-231, E-Mail: heidemarie.simon@ icomedias.com, Internet: www.icomedias.com.

\section{Mitarbeiterportal}

MyGSB heißt ein vorkonfiguriertes Mitarbeiterportal der MATERNA GmbH. Es wurde auf Basis der Content-Management-Lösung Government Site Builder (GSB) und der Formularmanagement-Lösung FormsForWeb der Firma Lucom realisiert. Hierbei handelt es sich um zwei Basiskomponenten der Initiative BundOnline. Das FMS ermöglicht die einfache Abbildung gängiger Geschäftsprozesse im Mitarbeiterportal, zum Beispiel das elektronische Beantragen und Genehmigen von Urlaubs- und Dienstreiseanträgen. MyGSB lässt sich ferner in gängige Portal-Server integrieren und bietet damit die Basis für komplexe Intranet-Lösungen und Mitarbeiterportale. Die Lösung basiert auf typischen Anforderungen, die an ein Intranet in der Verwaltung gestellt werden.

Weitere Informationen: MATERNA GmbH, Christine Siepe, Voßkuhle 37, 44141 Dortmund, Telefon: (0231) 55 99-168, EMail: Christine.Siepe@Materna.de,http://www.materna.de/presse.

\section{Betrugsbekämpfung im Internet}

Das Bundesinnenministerium und die Initiative D21, Europas größte Partnerschaft zwischen Politik und Wirtschaft, haben den Abschlussbericht der Projektgruppe »Effektive Betrugsbekämpfung « vorgestellt. Die Handlungsempfehlungen für Verbraucher, Wirtschaft und Verwaltung haben das Ziel, die Betrugsgefahr im Internet zu reduzieren. Der vom Bundesinnenministerium unterstützten Projektgruppe unter Leitung von Dr. Anja Bundschuh (Director Government Relations Europe bei eBay) gehörten Vertreter von Unternehmen und Verbänden, die u.a. in den Bereichen OnlineHandel und Online-Banking aktiv sind, sowie Mitarbeiter von Strafverfolgungsbehörden, Ministerien und Präventionsgremien an.

Die Informationen sowie der Abschlussbericht sind im Internet abrufbar unter www.bmi.bund.de und www.initiatived21.de.

\section{Symposium Personalbeurteilung}

Fragen der Beurteilung von Mitarbeitern/innen stoßen derzeit bei Praktikern des öffentlichen Sektors auf reges Interesse. Hintergrund hierfür ist der neue Tarifvertrag TVöD (Nachfolger des BAT), wel- 